Jörg Lindenmeier

\title{
Das Management von Anspruchsgruppen in öffentlichen Organisationen: Ein durch Dieter K. Tscheulin vorangetriebenes Forschungsfeld
}

\section{Management von Anspruchsgruppen in öffentlichen Organisationen}

In den 1980er Jahren haben sich unter dem Schlagwort "New Public Management” Reformbewegungen herausgebildet, welche die Einführung und Umsetzung managerialistisch geprägter Konzepte in öffentliche Verwaltungen und Betrieben vorsehen. Zum einen umfassen diese Vorschläge Konzepte der Binnenreformierung. Hierzu zählen bspw. die Implementierung von Globalbudgets oder aber Dezentralisierungs- und Disaggregationsanstrengungen auf der Ebene der Organisationstruktur öffentlicher Verwaltungen und Behörden (vgl. z. B. Thom/ Ritz 2008). Zum anderen wurden auch Instrumente der Verwaltungsmodernisierung entwickelt, welche stärker nach außen gerichtet sind und sich nicht zuletzt in einer stärker ausgeprägten Kundenund Wettbewerbsorientierung niederschlagen sollten (vgl. z. B. Schedler/Proeller 2011).

Die Kundenorientierung sowie die Wettbewerbsorientierung repräsentieren Phänomene, welche auch als Dimensionen des strategischen Konzeptes der Marktorientierung angesehen werden. In ihrer klassischen Definition umschließt die Marktorientierung auch die Dimension der interfunktionalen Koordination, die durch die abteilungsübergreifende Koordination und das Teilen von Marktinformationen innerhalb der Organisation beschrieben werden kann (vgl. z. B. Narver/Slater 1990). Die Idee der Marktorientierung besitzt insbesondere in der Marketing- aber auch der Organisationsforschung seit längerer Zeit eine hohe Relevanz (vgl. bspw. Narver/ Slater 1990; Kohli/Jaworski 1990; Deng/Dart 1994). Verschiedene Einzelstudien konnten einen positiven Effekt der Marktorientierung auf die Performance erwerbswirtschaftlicher Unternehmen nachweisen (z. B. Jaworski/Kohli 1993 oder Morgan et al. 2009). Allerdings konnte auch gezeigt werden, dass bspw. das Wettbewerbsumfeld (Slater/Narver 1994) oder aber Variationen in den grundlegenden Strategietypen (Matsuon/Mentzer 2000) die Beziehung zwischen Marktorientierung und Performance moderieren und hier dementsprechend nicht naiv von einem linearen Funktionsverlauf ausgegangen werden kann. Metastudien wie die von Shoham/Rose/ Kropp (2005) oder Ellis (2006) konnten nichtsdestotrotz einen im Allgemeinen bestehenden, positiven Effekt der Marktorientierung auf die Performance erwerbswirtschaftlicher Unternehmen untermauern. 
In Anlehnung an die grundlegende Philosophie des „New Public Management“ wurde und wird eine Orientierung am Konzept der Marktorientierung auch für öffentliche Organisationen vorgeschlagen. Allerdings lassen Arbeiten wie bspw. die von Walker et al. (2011) Zweifel an der Zweckmäßigkeit des stark erwerbswirtschaftlich geprägten Konzepts aufkommen. Die Autoren zeigen, dass eine höhere Marktorientierung von Kommunalverwaltungen zwar zu einer höheren wahrgenommenen Zufriedenheit bei den Bürgern führt. Auf anderen Ebenen konnten jedoch keine positiven Effekte der strategischen Neuausrichtung öffentlicher Organisationen nachgewiesen werden. Die bestehenden Zweifel im Hinblick auf die Sinnhaftigkeit der Marktorientierung in öffentlichen Organisationen geht auch konform mit der generellen Kritik, welche am Konzept des „New Public Management“ geübt wird (vgl. für viele z. B. Diefenbach 2009). Cervera et al. (2000) merken in diesem Zusammenhang an, dass öffentliche Verwaltungen nicht ausschließlich als Produzenten von Dienstleistungen betrachtet werden dürfen. Vielmehr müsse die Leitung öffentlicher Verwaltungen in ihrem Handeln auch Gesetzen und Verordnungen entsprechen, alle Bürger gleich und gerecht behandeln sowie einen diskriminierungsfreien Zugang zu öffentlichen Leistungsangeboten gewährleisten. Nur so könne die öffentliche Verwaltung ihre demokratischen Pflichten erfüllen und den Bürgern die notwendigen Entfaltungsräume ermöglichen.

Vor dem Hintergrund dieser Kritik an einer direkten und ungefilterten Umsetzung des Konzeptes der Marktorientierung im öffentlichen Sektor schlagen Cervera et al. (2000) Anpassungen des primär erwerbswirtschaftlich ausgerichteten Ansatzes vor. Die Autoren heben in diesem Zusammenhang heraus, dass bei einer strategischen Umorientierung öffentlicher Organisationen in Richtung einer stärkeren Marktorientierung ein besonderes Augenmerk auf die Generierung von Informationen über Bürger, Wettbewerber und sonstige organisationsexterne Entitäten sowie die Verbreitung der generierten Informationen innerhalb der Organisation gelegt werden sollte. Aufbauend auf diesen Schritten der Marktorientierung in öffentlichen Organisationen sollen Produkte und Dienstleistungen entwickelt, bepreist, beworben und vertrieben werden. Typische Marketingstrategien wie bspw. Segmentierungs- und Targeting-Ansätze können hierbei - unter Berücksichtigung der spezifischen Ziele öffentlicher Unternehmen - Anwendung finden. Konformgehend mit Jaworski/Kohli (1993) finden die oben erwähnten Dimensionen der Kunden- und Wettbewerbsorientierung in der Konzeptionierung von Cervera et al. (2000) keine explizite Berücksichtigung.

Interessanterweise konnte eine Metaanalyse von Cano et al. (2004) eine stärkere positive Wirkung der Marktorientierung auf die Performance von nicht-gewinnorientierten Organisationen als auf die Performance von gewinnorientierten Unternehmen zeigen. Diese Befundlage kann als starkes Argument für die Marktorientierung als grundlegende strategische Ausrichtung für gemeinnützige und öffentliche Organisationen angesehen werden. Liao/Foreman/Sargeant (2001) kritisieren allerdings eine (naive) Übertragung des Konzepts der Marktorientierung auf nicht-gewinnorientierte Organisationen und schlagen die Entwicklung eines Konzeptes der sogenannten „Societal Orientation“ vor. Dieser Ansatz bezieht sich hierbei zwar insbesondere auf gemeinnützige Organisationen; kann aber durchaus auch Impulse für den Bereich der öffentlichen Organisationen geben. Vergleichbar mit dem Konzept der klassischen Marktorientierung umfasst dieser Denkansatz die abteilungsübergreifende Koordination sowie die Wettbewerbsfokussierung. Letzterer wird allerdings durch den Denkansatz der Kollaboration erweitert. Kolla- 
boration kann sich hierbei auf die Zusammenarbeit mit anderen gemeinnützigen Organisationen oder aber auch öffentlichen Einrichtungen und erwerbswirtschaftlichen Unternehmen beziehen (bspw. im Rahmen des Sponsorings). „Private Public Partnerships“ wären ein Beispiel einer Kollaborationsform, die für den öffentlichen Sektor bedeutend ist. Genauso wie Rodrigues et al. (2012) berücksichtigen Liao et al. (2001), neben Effizienz und Effektivität als ökonomische Erfolgskategorien, den gesellschaftlichen Nutzen als ein Maß des nicht-ökonomischen Erfolgs. Einer der wesentlichen Unterschiede zwischen der klassischen Marktorientierung von erwerbswirtschaftlichen Unternehmen und dem Konzept der „Societal Orientation“" von Liao et al. (2001) liegt darin, dass nicht nur eine reine Kundenorientierung, sondern eine breiter angelegte Stakeholder- bzw. Anspruchsgruppenorientierung angestrebt werden soll. In Übereinstimmung mit dem Gedanken der Orientierung an multiplen Anspruchsgruppen wird die Kongruenz der organisationalen Ziele mit den Zielen der Anspruchsgruppen dementsprechend als ein Antezedent der „Societal Orientation“ angesehen. An dieser Stelle kann herausgestellt werden, dass dem Ansatz der „Public Corporate Governance“, welche als Weiterentwicklung und/oder Ergänzung des „New Public Managements“ charakterisiert werden kann, die Fokussierung auf verschiedene Stakeholder ebenfalls inhärent ist (vgl. z. B. Bovaird 2005). Die vorgeschlagene Berücksichtigung multipler Anspruchsgruppen wird von einer gewissen Zahl an Studien zur Marktorientierung in öffentlichen Organisationen aufgegriffen (bspw. Paarlberg 2007; Mainardes/Raposo/Alves 2014), zumeist werden hierbei allerdings primär einzelne Anspruchsgruppen wie bspw. Kunden bzw. Bürger (bspw. Schedler/Summermatter 2007) oder Mitarbeitende (bspw. Flavian/Lozarno 2007) betrachtet. Als weitere Dimensionen der „Societal Orientation“Konzeption wird von Liao et al. (2000) die Ansprechbarkeit und Reaktionsfähigkeit von Organisationen miteingeschlossen, welche sich auf verschiedene Stakeholder und deren Bedürfnisse bezieht und demnach eng mit der Anspruchsgruppenorientierung verwoben ist. Cervera et al. (2000) schlägt demgemäß im Rahmen der (Weiter-) Entwicklung öffentlicher Programme und Leistungsportfolios ein „Response Design“ sowie eine „Response Implementation“ vor, welche es öffentlichen Organisationen ermöglichen soll, auf die Wünsche und Bedürfnisse von Bürgern und anderer Anspruchsgruppen schnell und proaktiv einzugehen. Die entsprechenden Ansätze basieren hierbei entscheidend auf ,,marktbezogene“ Informationen und setzen demgemäß die Implementierung von Systemen der empirischen „Markt“-Forschung voraus.

Die skizzierte Hinwendung hin zu einer stärkeren Markt- und/oder Anspruchsgruppenorientierung in öffentlichen Organisationen wird auch durch verschiedene wissenschaftliche Fachbeiträge in der Zeitschrift für öffentliche und gemeinwirtschaftliche Unternehmen (ZögU) reflektiert. So diskutiert Dietrich (2007) in einer konzeptionellen Arbeit den Sinn und Zweck von Marktstrategien für kommunale Dienstleister. Analysen und Untersuchungen, welche inhaltlich nahe an der klassischen Marktorientierung lokalisiert sind, finden sich ferner zum einen in den Papieren von Drevs (2013), Hausmann (2006), Hausmann/Pöllmann (2014) sowie Jobst/Boerner (2014). Die entsprechenden Papiere fokussieren hierbei jeweils den Bereich der öffentlichen Kulturbetriebe. Zum anderen stellen die Arbeiten von Dietrich/Gapp (2005) sowie Tscheulin et al. (2001) die Wahrnehmungen und Präferenzen von Patienten öffentlicher Krankenhäuser in den Fokus der Betrachtung. Scherhag/Boenigk (2010) nehmen in ihrer auf gemeinnützige Organisationen bezogenen Studie darüber hinaus Spender und ihre langfristige Bindung im Rahmen einer ,Relationship Fundraising“-Konzeption in den Blick. 
In einem weiteren verwandten Literaturstrang wird in der ZögU mit den Mitarbeitenden eine ebenfalls wichtige Anspruchsgruppe von öffentlichen Organisationen adressiert. In zwei aktuellen Papieren betrachten Andersch et al. (2017) sowie Vogel/Keppeler/Papenfuß (2018) die Phänomene der Arbeitgeberattraktivität sowie der Public Service Motivation aus der Perspektive öffentlicher Verwaltungen. Nienaber/Tietmeyer (2010) analysieren die Unterschiede in der Arbeitsmotivation von Mitarbeitenden in gemeinnützigen und gewinnorientierten Organisationen. Hirsch et al. (2009) entwickeln Konzepte für Anreizsysteme von Militärangehörigen. Saliterer/ Korac (2013) eruieren den Effekt persönlicher Faktoren auf die Innovationstätigkeit öffentlicher Verwaltungen. Hausmann/Süß (2013) diskutieren ferner Fragestellungen des Diversity Managements. Esslinger (2006) fokussiert in einer tendenziell eher auf das Controlling und die Steuerung gemeinnütziger Organisationen ausgerichteten Arbeit das Spannungsfeld zwischen hauptamtlich und freiwillig tätigen Mitarbeitenden.

In weiteren ZögU-Beiträgen analysieren Drescher/Schäfer (2015 und 2017) sowie Wessel (2016) Instrumente einer partizipativen Finanzierung von öffentlichen Leistungen. Hainzer/ Stötzer/Ellmer (2014) fokussieren mit „Crowdfunding“-basierten Bürgerkrediten eine ähnlich gelagerte Thematik mit Bezug zu den Bürgern als wichtige Stakeholdergruppe. Weitere Arbeiten, welche unternehmensethisch relevante Themen bearbeiten und hierbei ebenfalls die Bürger als breite und diverse Anspruchsgruppe berücksichtigen, finden sich bei Drevs et al. (2012), Poller et al. (2014) und Lindenmeier et al. (2009). In dem verwandten Bereich der „Public Corporate Governance“" veröffentlichen Andeßner (2016), Brämer et al. (2016) sowie Greiling/ Häusler (2016) Papiere mit direktem Bezug zum Thema des Managements von Anspruchsgruppen.

\section{Das Wirken von Dieter K. Tscheulin im Bereich des Management von Anspruchsgruppen in öffentlichen Organisationen (und darüber hinaus)}

Neben den am Ende des vorausgegangenen Kapitels erwähnten Beiträgen wurden in den letzten Jahren zwei Beihefte der ZögU mit einem expliziten Fokus auf die Markt- und Anspruchsgruppenorientierung veröffentlicht. Während sich das erste dieser beiden Beihefte mit der Thematik des „Public und Nonprofit Marketing“ beschäftigt, umfasst das zweite Beiheft Papiere zur Akzeptanz von Aktivitäten öffentlicher Organisationen in verschiedenen Anspruchsgruppen. Es stellt hierbei keinen Zufall dar, dass die Beihefte von Dieter K. Tscheulin (mit-) herausgegeben wurden. Seine wissenschaftlichen Arbeiten hatten und haben einen signifikanten Effekt auf die Forschung zum Management von Anspruchsgruppen in öffentlichen Organisationen.

Nach dem Studium der Betriebs- und Volkswirtschaftslehre in Gießen und Kiel mit Auslandsstudienaufenthalten in Italien und Schweden beginnt Dieter K. Tscheulin eine Promotion an der WHU Koblenz sowie Universität Lüneburg. Seine Dissertation beschäftigt sich mit dem Thema der optimalen Produktgestaltung mit Hilfe der Conjointanalyse sowie der „Analytic Hierarchy Process"-Methodik. Wie in den vorangegangenen Ausführungen herausgestellt wurde, stellt die Generierung und Verarbeitung von marktbezogenen Informationen sowie die der Marketing- 
wissenschaft inhärente Betrachtung (konsumenten-) psychologischer Konzepte eine Grundvoraussetzung für die Umsetzung eines Managements von Anspruchsgruppen in öffentlichen Organisationen dar. Die Forschungen Dieter K. Tscheulins zeigen, wie individuelle Präferenzen valide messbar gemacht werden können. Indem sie aufzeigen, wie eine bedürfnisorientierte Gestaltung von Leistungsangeboten vonstattengehen kann, gaben diese Arbeiten damit auch einen entscheidenden Impuls für die anspruchsgruppenorientierte „Public Management“-Forschung. Die entsprechenden Informationen sind essentiell für die durch das „New Public Management“ empfohlene stärkere Markt- und Wettbewerbsorientierung öffentlicher Organisationen.

Im Jahre 1990 folgt Dieter K. Tscheulin einem Ruf auf eine Junior-Professur an der Université Notre-Dame de la Paix Namur in Belgien. In der belgischen Zeit richteten sich die Forschungsinteressen Dieter K. Tscheulins stärker auf das Management von Dienstleistungen hin aus. Vor diesem Hintergrund stellt es eine konsequente Entwicklung dar, dass er ein langjähriges Mitglied des wissenschaftlichen Beirats (WBR) des Bundesverbandes öffentliche Dienstleistungen (bvöd) ist. Dort ist Dieter K. Tscheulin insbesondere für die Themenfelder des Gesundheitsmanagements, der öffentlichen Betriebswirtschaftslehre und des Nonprofit Managements zuständig und fördert in deren Kontext den Transfer von neuesten wissenschaftlichen Erkenntnissen in die Praxis öffentlicher Versorgungs- und Dienstleistungsunternehmen. Eng mit dieser Tätigkeit verbunden ist seine Angehörigkeit zum Kreis der Herausgeber der ZögU, welche eines der Publikationsorgane des bvöd repräsentiert. Die weiter oben aufgeführten Papiere zum Management von Anspruchsgruppen in öffentlichen Organisationen, welche über die Jahre in der ZögU publiziert wurden, können mittelbar oder unmittelbar auf das Wirken Dieter K. Tscheulins im Herausgeberkreis zurückgeführt werden.

Im Jahre 1993 nimmt Dieter K. Tscheulin einen Ruf an die Albert-Ludwigs-Universität Freiburg an, welchem weitere Rufe folgen. Trotz dieser Rufe bleibt er in Freiburg und besetzt dort eine C4-Professur für Marketing und Gesundheitsmanagement. Neben der bereits gewürdigten Bedeutung des Marketings für das anspruchsgruppenorientierte Management öffentlicher Organisationen repräsentiert der zweite Teil der Denomination einen wichtigen Subsektor der öffentlichen Wirtschaft. Dieter K. Tscheulin bearbeitet die Thematik des Gesundheitsmanagements bereits in den 1990er Jahren als einer der Pioniere im deutschsprachigen Raum. Seine intensive Forschungstätigkeit wird durch eine Vielzahl wissenschaftlicher Publikationen reflektiert, welche in großen Teilen die Idee der Anspruchsgruppenorientierung öffentlicher und gemeinnütziger Organisationen in der Gesundheitswirtschaft zugrunde liegt. Als Kommissionsvorsitzender kann Dieter K. Tscheulin ab 2004 ferner die Bedeutung des Managements im Gesundheitswesen in der Wissenschaftlichen Kommission Öffentliche Betriebswirtschaftslehre (WK ÖBWL) des Verbandes der Hochschullehrer für Betriebswirtschaft e.V. (VHB) signifikant stärken. In der Lehre kann er ein Wahlfach „Gesundheitsmanagement“" im volkswirtschaftlichen Lehrangebot der Albert-Ludwigs-Universität sowie einen Kontaktstudiengang „Health Care Management" an der Freiburger Akademie für Universitäre Weiterbildung (FRAUW) etablieren.

Während seiner Zeit als Dekan der Wirtschafts- und Verhaltenswissenschaftlichen Fakultät der Albert-Ludwigs-Universität Freiburg kann Dieter K. Tscheulin gegen Widerstand ein betriebswirtschaftliches Bachelorprogramm mit einer Spezialisierung auf das Public und Non-Profit Management einführen und somit die Bedeutung des Faches „Management öffentlicher Organisationen“ in der deutschen Universitätslandschaft massiv stärken. Im Zuge dieser Einführung 
kann eine einschlägige W3-Professur sowie eine ebenfalls einschlägige W1-Professur („Tenure Track") eingerichtet werden. Zum Wintersemester 2016/2017 folgt ein konsekutives Masterprogramm im Zuge dessen eine zweite W3-Professur im Bereich des Public und Non-Profit Managements eingerichtet wird. Im Rahmen der betriebswirtschaftlichen Lehrveranstaltungen an der Albert-Ludwigs Universität Freiburg werden den Studierenden die Instrumente und Werkzeuge eines anspruchsgruppenorientierten Managements öffentlicher und gemeinnütziger Organisationen vermittelt.

\section{Management von Anspruchsgruppen in öffentlichen Organisationen}

Das vorliegende Heft schließt an der von Dieter K. Tscheulin betriebenen bzw. initiierten Forschung als auch seinen sonstigen Aktivitäten an und stellt das Management von Anspruchsgruppen in öffentlichen Organisationen in das Zentrum der Betrachtung. Die einzelnen Beiträge stammen dabei von Autoren, welche Dieter K. Tscheulin entweder unmittelbar als akademischer Lehrer oder mittelbar über die Rezeption seiner Forschungstätigkeit beeinflusst hat.

Konformgehend mit der oben erwähnten Studie von Rodrigues et al. (2018) wird im vorliegenden Beiheft zwischen einer nach innen und außen gerichteten Anspruchsgruppenfokussierung unterschieden. Dabei werden einerseits Bürger, Kunden öffentlicher Organisationen und Politiker als externe Anspruchsgruppen berücksichtigt (Znotka et al.; Liberatore et al.; Eggert et al.; Korac et al.; Saliterer/Albrecht; Seemann/Jahed; Trautwein et al.). Im Gegensatz zur rein erwerbswirtschaftlich orientierten Auslegung des Konzeptes der Marktorientierung soll hierbei jedoch nicht eine Maximierung von Gewinnen auf Basis von Kundenpräferenzen adressiert werden. Vielmehr wird hierbei insbesondere eine Verbindung zwischen den individuellen Präferenzen und Bedürfnissen bestimmter Anspruchsgruppen und dem gesetzlichen oder politischen Auftrag öffentlicher Organisationen sowie der Effektivität und Effizienz der Erfüllung öffentlicher Aufgaben hergestellt.

Die Papiere von Znotka et al., Liberatore et al., Saliterer/Albrecht, Seemann/Jahed und Trautwein et al. beziehen sich auf Bürger als externe Anspruchsgruppen. Der Beitrag von Znotka et $a l$. ist im Bereich der Planung und Steuerung von Geschäftsmodellen öffentlicher Organisationen angesiedelt. Hierbei wird in einer Studie mit Bezug zum Gesundheitswesen untersucht, welche Aspekte der Tätigkeit öffentlicher Unternehmen wesentlich zur Schaffung öffentlicher Werte beitragen. Aufbauend auf einer qualitativen Untersuchung identifizieren die Autoren drei „Public Value“-Kategorien, auf deren Basis Manager in öffentlichen Unternehmen Leistungsangebote anspruchsgruppenorientiert planen können. Seemann/Jahded liefern ein Papier mit Relevanz für die öffentliche Wohnungswirtschaft. Die Autorinnen betrachten mit dem Konzept der Baugruppen einen innovativen Ansatz der Vergabe und Entwicklung städtischer Liegenschaften. Aufbauend auf einem Innovationsakzeptanzmodell identifizieren die Autorinnen verschiedene Treiber und Hemmschuhe der Neigung von Bürgern zu Baugruppen, welche bspw. kommunale Wohnungsbaugesellschaften oder Ämter für Liegenschaften- und Wohnungswesen im Rahmen einer anspruchsgruppenorientierten Gestaltung von Baugruppenkonzepten berück- 
sichtigen können. Saliterer/Albrecht untersuchen den Effekt unterschiedlicher Informationsquellen sowie weiterer Variablen auf die Glaubwürdigkeit von Informationen über die Performance öffentlicher Unternehmen. Auf Basis von Ergebnissen einer großzahligen experimentellen Studie können die Autorinnen zeigen, dass Informationen besonders dann glaubwürdig sind, wenn diese aus der privaten Gesellschaftssphäre stammen und die Informationsquelle als unabhängig angesehen werden kann. Das Papier von Liberatore et al. ist auf dem Themenfeld der öffentlichen Gesundheitskampagnen angesiedelt und betrachtet, wie eine spezifische Anspruchsgruppe öffentlicher Gesundheitsbehörden zur Verbreitung wichtiger Informationen zur Gesundheitsprävention motiviert und damit zu einer Ko-Produktion eines öffentlichen Gutes beitragen kann. Darüber hinaus zeigt das Papier einen Ansatz zur Quantifizierung der Effekte der entsprechenden Ko-Produktions-Aktivitäten. Indem sie die Gefahr von sozial erwünschten Antworten in Befragungsstudien von öffentlichen Organisationen analysieren, setzen Trautwein et al. mit ihrer methodischen Arbeit noch stärker als die anderen Arbeiten an der Marktinformationskomponente der Markt- bzw. Anspruchsgruppenorientierung an. Aufbauend auf experimentellen Daten wird u. a. gezeigt, dass es im Rahmen von Markt- und Meinungsforschungsstudien sinnvoll ist, die Trägerschaft der Studie durch öffentliche Institutionen als vertrauensstiftende gegenüber den befragten Personen transparent zu machen.

Korac et al. fokussieren Politiker und Politikerinnen als externe Anspruchsgruppe. Unter Berücksichtigung des Instruments des „Gender Budgeting“ wird hierbei im Rahmen einer qualitativen Analyse untersucht, wie die Mitglieder der Anspruchsgruppe geschlechtsspezifische Themen im öffentlichen Budgetprozess wahrnehmen. Die Befragten stellen in ihren Antworten insbesondere die geringe Reaktionsfreudigkeit und die geringe aktive Partizipation administrativer Einheiten heraus. Beides deutet auf eine geringe Anspruchsgruppenorientierung in der öffentlichen Verwaltung hin. Die spieltheoretisch ausgerichtete Arbeit von Eggert et al. ist auf der Ebene von Nationalstaaten angesiedelt und schließt gewinnorientierte Unternehmen als Anspruchsgruppe öffentlicher Institutionen mit in die Betrachtung ein. Im Wettbewerb um mobile Unternehmensgewinne können Nationalstaaten Steuersätze sowie Transparenzanforderungen steuern, um mit einem gezielten Management der Anreize dieser Anspruchsgruppen ihre Ziele zu erreichen. Im Fall eines wiederholten Spiels kann ein effizientes Transparenzniveau dann erreicht werden, wenn eine Bestrafung für Verstöße gegen die Transparenzvorschriften glaubwürdig angedroht werden kann. Diese Befunde der Arbeit liefern öffentlichen Organisationen auf der nationalen und supranationalen Ebene wertvolle Hinweise für die Ausgestaltung der Transparenzanforderungen in internationalen Steuersystemen.

Andererseits nimmt das Beiheft mit den Mitarbeitenden auch eine wichtige interne Anspruchsgruppe in den Blick (Helmig et al.; Winter/Thaler/ Gross, Mayr/Boenigk). Eine Ausrichtung des Personalmanagements öffentlicher Organisationen an den Bedürfnissen der Anspruchsgruppe der Mitarbeitenden sollte hierbei in einer höheren Arbeitsmotivation sowie einer höheren Loyalität der Mitarbeitenden gegenüber ihrem Dienstherrn oder Arbeitgeber resultieren. Helmig et al. betrachten eine Gruppe von kulturell heterogenen Mitarbeitenden in einer internationalen öffentlichen Organisation. Im Rahmen einer quantitativen Studie untersuchen die Autoren, den Effekt der „Public Service Motivation“ auf das „Commitment“ der Mitarbeitenden. Abweichend von der bisherigen Befundlage können sie zeigen, dass die Person-OrganisationsPassung den unterstellten Effekt nicht mediiert. Der Beitrag von Mayr/Boengik stellt die Wis- 
sensgenerierung, -speicherung und -weitergabe in Projektteams in den Fokus der Betrachtung. Hierbei setzen die Forschungen an dem gruppenpsychologischen Konzept der transaktiven Speichersysteme an. Unter Berücksichtigung von Daten aus dem Bereich der öffentlichen und gemeinnützigen Organisationen konnte eine Mehrebenenanalyse zeigen, dass transformationale Führungsstile einen positiven Effekt auf die Generierung abrufbaren Gruppenwissens haben können. Ferner konnte auch ein positiver Effekt der transaktiven Speichersysteme auf die individuelle Mitarbeitendenzufriedenheit in den einzelnen Organisationen festgestellt werden. Winter et al. fokussieren ebenfalls Mitarbeitende öffentlicher Organisationen und untersuchen, welches Verhalten Mitarbeitende im Krankheitsfall an den Tag legen. Insbesondere werden die Phänomene des Absentismus sowie des Präsentismus betrachtet. Die empirische Arbeit zeigt eine positive Korrelation zwischen beiden Phänomenen und widerspricht hierbei der gemeinhin unterstellten Substitutionsbeziehung zwischen den beiden Verhaltensweisen. Aufbauend auf den Studienergebnissen empfehlen die Autorinnen, der Anspruchsgruppe der kranken Mitarbeitenden gleichermaßen Anti-Absentismus- und Anti-Präsentismus-Programme anzubieten. Ferner werden insbesondere auch die negativen Wirkungen einer Präsentismus-Kultur herausgestellt.

Die Papiere dieses Hefts beschäftigen sich aus unterschiedlichen Perspektiven mit der Thematik des anspruchsgruppenorientierten Management in öffentlichen Organisationen. Neben den unterschiedlichen Anspruchsgruppen und theoretischen Zugängen zu dem Forschungsfeld sei an dieser Stelle insbesondere auch der breite Methodenmix der vorliegenden Arbeiten erwähnt, der von qualitativen Studien über experimentelle und fragebogenbasierten Forschungsdesigns bis hin zu spieltheoretischen Ausarbeitungen reicht. Mögen die Beiträge des vorliegenden Hefts zu einer kritischen Reflektion über den Sinn und Zweck sowie die zielgerichtete Ausgestaltung eines Managements von Anspruchsgruppen in öffentlichen Organisationen anregen. Insbesondere stellt sich hierbei die Frage, inwieweit der „Bogen der Marktorientierung“ in öffentlichen und gemeinwirtschaftlichen Unternehmen gespannt werden kann. Es bleibt insbesondere zu hoffen, dass die Impulse, welche Dieter K. Tscheulin dem im Heft bearbeiteten Forschungsfeld gegeben hat, weiterhin Widerhall finden und aktuell aktive sowie zukünftige Forscher und Forscherinnen dementsprechend an seinem wissenschaftlichen Oeuvre anknüpfen werden können.

\section{Referenzen}

Andersch, H., Liberatore, F., Hurst, A., Kern, A., Lindenmeier, J. und Wieselhuber, S. (2016), Reputation öffentlicher Arbeitgeber-Validierung eines Messkonzeptes am Beispiel von Mitarbeitenden einer deutschen Gemeindeverwaltung, in: Zeitschrift für öffentliche und gemeinwirtschaftliche Unternehmen, 39. Jg., Heft 4, S. 325-341.

Andeßner, R. C. (2016), Civic engagement and local public governance in Austria-Traditions, current challenges and possible future paths, in: Zeitschrift für öffentliche und gemeinwirtschaftliche Unternehmen, 39. Jg., Heft 1-2, S. 41-52.

Bovaird, T. (2005), Public governance: balancing stakeholder power in a network society, in: International Review of Administrative Sciences, 71 Jg., Heft 2), S. 217-228.

Brämer, P., Gischer, H., Pfingsten, A. und Richter, T. (2010), Der öffentliche Auftrag der deutschen Sparkassen aus der Perspektive des Stakeholder-Managements, in: Zeitschrift für öffentliche und gemeinwirtschaftliche Unternehmen, 33. Jg., Heft 4, S. 311-332. 
Cano, C. R., Carrillat, F. A. und Jaramillo, F. (2004), A meta-analysis of the relationship between market orientation and business performance: evidence from five continents, in: International Journal of research in Marketing, 21. Jg., Heft 2, S. 179-200.

Cervera, A., Mollá, A. und Calderon, H. (2000), Market orientation: A framework for public institutions, in: Journal of Nonprofit und Public Sector Marketing, 7. Jg., Heft 1, S. 3-23.

Deng, S. und Dart, J. (1994), Measuring market orientation: a multi-factor, multi-item approach, in: Journal of Marketing Management, 10. Jg., Heft 8, S. 725-742.

Diefenbach, T. (2009), New public management in public sector organizations: the dark sides of managerialistic 'enlightenment', in: Public Administration, 87. Jg., Heft 4, S. 892-909.

Dietrich, M. (2007), Marktstrategien für kommunale Dienstleistungsanbieter im Wettbewerb. ZögU Zeitschrift für öffentliche und gemeinwirtschaftliche Unternehmen, $30 \mathrm{Jg}$., Heft 1, S. 31-51.

Dietrich, M. und Gapp O. (2005), Qualitätsinformationen von Krankenhäusern: Eine Untersuchung ihrer Relevanz und Anforderungen aus Patientensicht, in: Zeitschrift für öffentliche und gemeinwirtschaftliche Unternehmen, 28. Jg. Heft 3, 211-233.

Drevs, F. und Renner, S. (2013), Der Einfluss staatlicher Zuschüsse auf Zahlungsbereitschaften für Erzeugnisse aus multifunktionaler Landwirtschaft. In Akzeptanz des öffentlichen Ausgabeverhaltens, hrsg. Martin Dietrich und Dieter K. Tscheulin, ZögU Beihfet 42, Baden-Bade, S. 84-104.

Drescher, S., und Schaefer, C. (2015), Über das partizipative Moment im Rahmen der Finanzierung öffentlicher Unternehmen, in: Zeitschrift für öffentliche und gemeinwirtschaftliche Unternehmen, Jg. 38, Heft 1, S. 3-20.

Drescher, S., und Schaefer, C. (2017), Partizipative Finanzierung als Instrument zur Einbindung der Bürger in eine präferenzgerechte und wohlfahrtsorientierte Leistungserbringung-Theoretischer Rahmen und konzeptionelle Ausgestaltung am Beispiel der BürgerEnergie Rhein-Sieg e.G., in: Zeitschrift für öffentliche und gemeinwirtschaftliche Unternehmen, Jg. 40, Heft 1, S. 29-49.

Ellis, P. D. (2006), Market orientation and performance: A meta-analysis and cross-national comparisons, in: Journal of Management Studies, Jg. 43, Heft 5, S. 1089-1107.

Esslinger, A. S. (2006), Zielorientiertes Management in sozialen Einrichtungen im Spannungsfeld zwischen Haupt-und Ehrenamt, in: Zeitschrift für öffentliche und gemeinwirtschaftliche Unternehmen, 29. Jg., Heft 2, S. 147-164.

Flavián, C., und Lozano, J. (2007), Market orientation of Spanish public universities: A suitable response to the growing competition, in: Journal of Marketing for Higher Education, 17. Jg., Heft 1, S. 91-116.

Greiling, D., und Häusler, E. (2016), Stakeholder accountability of Austrian and German health insurance funds, in: ZögU Zeitschrift für öffentliche und gemeinwirtschaftliche Unternehmen, 39. Jg., Heft 1-2, S. 143-162.

Hainzer, M., Stötzer, S., und Ellmer, M. (2014), Crowdfunding-basierte Bürgerkreditmodelle in Kommunen. ZögU Zeitschrift für öffentliche und gemeinwirtschaftliche Unternehmen, 37. Jg., Heft 1-2, S. 54-72.

Hausmann, A. (2006), Preispolitische Optionen zur Erlösoptimierung von Museumsbetrieben, in: Zeitschrift für öffentliche und gemeinwirtschaftliche Unternehmen, 29. Jg., Heft 3, S. 241-258.

Hausmann, A., und Pöllmann, L. (2014), Nutzer und Nutzung der Social Media-Profile von Theatern-Ergebnisse einer empirischen Untersuchung auf Facebook, in: Zeitschrift für öffentliche und gemeinwirtschaftliche Unternehmen, 37. Jg., Heft 1-2, S. 73-87.

Hausmann, A., und Süß, S. (2012), Personelle Vielfalt in der Kultur. Zur Bedeutung von Diversity-Management für Theaterbetriebe, in: Zeitschrift für öffentliche und gemeinwirtschaftliche Unternehmen, 35. Jg., Heft 4, S. 401-421.

Hirsch, B., Schmiedke, M., und Reemts, S. (2009), Anreizsysteme für Soldaten der Bundeswehr-Theoretische Analyse und Gestaltungsempfehlungen, in: Zeitschrift für öffentliche und gemeinwirtschaftliche Unternehmen, 32. Jg., Heft 1, S. 19-43.

Jobst, J., und Boerner, S. (2015), „Und wie fanden Sie es heute Abend?“ Eine empirische Analyse des Zuschauerurteils im Sprechtheater, in: Zeitschrift für öffentliche und gemeinwirtschaftliche Unternehmen, 37. Jg., Heft 4, S. 253-270.

Liao, M. N., Foreman, S., und Sargeant, A. (2001), Market versus societal orientation in the nonprofit context, in: International Journal of Nonprofit and Voluntary Sector Marketing, 6. Jg., Heft 3, S. 254-268.

Mainardes, E. W., Raposo, M., und Alves, H. (2014), Universities need a market orientation to attract non-traditional stakeholders as new financing sources in: Public Organization Review, 14. Jg., Heft 2, S. 159-171.

Matsuno, K., und Mentzer, J. T. (2000), The effects of strategy type on the market orientation-performance relationship, in: Journal of marketing, 64. Jg., Heft 4, S. 1-16.

Narver, J. C., und Slater, S. F. (1990), The effect of a market orientation on business profitability, in: Journal of Marketing, 54. Jg., Heft 4, S. 20-35.

Nienaber, A. M., und Tietmeyer, J. (2010), Wie sollten Non-Profit Organisationen ihre Mitarbeiter vertrauensvoll motivieren? Eine vergleichende Analyse bei gemeinnützigen und renditeorientierten Unternehmen, in: Zeitschrift für öffentliche und gemeinwirtschaftliche Unternehmen, 33. Jg. Heft 4, S. 333-353.

Paarlberg, L. E. (2007), The impact of customer orientation on government employee performance, in: International Public Management Journal, 10. Jg., Heft 2, S. 201-231.

Rodrigues, A. P., und Carlos Pinho, J. (2012), The impact of internal and external market orientation on performance in local public organisations, in: Marketing Intelligence und Planning, 30. Jg., Heft 3, S. 284-306. 


\section{Jörg Lindenmeier}

Saliterer, I., Korac, S. (2013), Drivers of local government innovation - do personal factors really matter?, in: Entrepreneurship in the public sector, hrsg. von Peter Eichhorn und Dorothea Greiling, ZögU Beiheft 43, BadenBaden, S. 148-160.

Schedler, K., und Proeller, I. (2011), New Public Management, 5. Aufl., Bern u. a.

Schedler, K., und Summermatter, L. (2007), Customer orientation in electronic government: Motives and effects, in: Government Information Quarterly, 24. Jg., Heft 2, S. 291-311.

Scherhag, C., und Boenigk, S. (2010), Relationship Fundraising: Stand der empirischen Forschung, theoretischer Bezugsrahmen und zukünftige Forschungsfelder, in: Zeitschrift für öffentliche und gemeinwirtschaftliche Unternehmen, 33. Jg., Heft 4, 354-367.

Shoham, A., Rose, G. M., und Kropp, F. (2005), Market orientation and performance: a meta-analysis, in: Marketing Intelligence und Planning, 23. Jg., Heft 5, S. 435-454.

Tscheulin, D. K., Helmig, B., und Moog, R. (2001), Die Ermittlung entscheidungsrelevanter Variablen der Wahl von Geburtskliniken als Basis eines effizienten Krankenhausmarketing. Zeitschrift für öffentliche und gemeinwirtschaftliche Unternehmen, 24. Jg., Heft 4, S. 451-468.

Thom, N., und Ritz, A. (2008), Public Management-Innovative Konzepte zur Führung im öffentlichen Sektor, 4. Aufl., Wiesbaden.

Kohli, A. K., und Jaworski, B. J. (1990), Market Orientation: The Construct, Research Propositions, and Managerial Implications, in: Journal of Marketing, 54. Jg., Heft 2, S. 1-18.

Vogel, R., Keppeler, F., und Papenfuß, U. (2018), Arbeitgeberattraktivität öffentlicher Unternehmen aus Sicht des Führungskräftenachwuchses der Verwaltung, in: Zeitschrift für öffentliche und gemeinwirtschaftliche Unternehmen, 40. Jg., Heft 4, S. 256-282.

Walker, R. M., Brewer, G. A., Boyne, G. A., und Avellaneda, C. N. (2011), Market orientation and public service performance: new public management gone mad?, in: Public Administration Review, 71. Jg., Heft 5, S. 707-717.

Wessel, F. (2016), Citizen financial participation schemes as part of New Public Governance, in: Zeitschrift für öffentliche und gemeinwirtschaftliche Unternehmen, 39. Jg. Heft 1-2, S- 53-64. 